\title{
Crystal structure of 2-(3,4-dimethylphenyl)-2-cyclohexene-1-one oxime, $\mathrm{C}_{14} \mathrm{H}_{17} \mathrm{NO}$
}

\author{
C. Meenakshi', V. Ramamoorthy', S. Muthusubramanian', S. Sivasubramanian', T. Ziegler"I and J. Lex*.III \\ I Madurai Kamaraj University, School of Chemistry, Madurai, 625021, India \\ "University of Tübingen. Institute of Organic Chemistry, D-72076. Tübingen, Germany \\ III University of Cologne, Institute of Organic Chemistry, Greinstraße 4. D-50939 Cologne, Germany
}

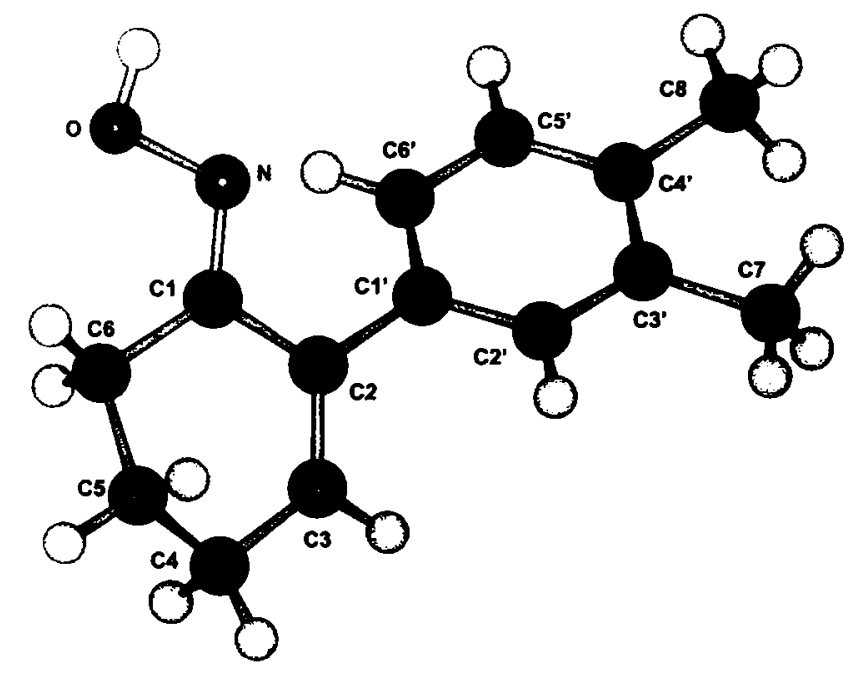

Abstract

$\mathrm{C}_{14} \mathrm{H}_{17} \mathrm{NO}$, triclinic, $P \overline{1}$ (No. 2), $a=6.731(1) \AA, b=8.757(1) \AA$, $c=11.348(1) \AA, \alpha=67.69(1)^{\circ}, \beta=80.71(1)^{\circ}, \gamma=79.29(1)^{\circ}$, $V=605.0 \AA^{3}, Z=2, R_{\mathrm{gt}}(F)=0.041, w R_{\mathrm{ref}}\left(F^{2}\right)=0.117, T=293 \mathrm{~K}$

\section{Source of material}

To a mixture of 1-(3,4-dimethyl phenyl)-cyclohexene $(62 \mathrm{~g})$, $n$-butyl nitrite $(35 \mathrm{~g})$ in glacial acetic acid $(65 \mathrm{ml})$ kept at $258 \mathrm{~K}$, a cold solution of $40 \mathrm{ml}$ of hydrochloric acid was added dropwise. The stirring was continued for a further period of 30 minutes and treated with $100 \mathrm{ml}$ of ice cold methanol. The precipitate was removed by filtration, washed twice with ice cold methanol $(25 \mathrm{ml})$ and dried to afford 1-(3,4-dimethylphenyl)-1-chloro-2-nitrosocyclohexane ( $57 \mathrm{~g}, 68 \%$ ); $\mathrm{mp} 288 \mathrm{~K}$ (dec.).

The above nitrosochloride $(28 \mathrm{~g})$ was suspended in pyridine $(60 \mathrm{ml})$ and warmed to $343 \mathrm{~K}$ whereupon the solid disolved. The mixture was kept at $343 \mathrm{~K}$ for a period of 10 minutes, cooled and acidified with dilute hydrochloric a cid. The resultant solid was filtered, washed with water and crystallized from ethanol to give 2-(3,4-dimethylphenyl)-2-cyclohexene-1-one oxime (17 g, $71 \%)$; mp $428 \mathrm{~K}$.

\section{Discussion}

The corresponding molecule parameters referring to those found in the two preceding derivatives $[1,2]$ have comparable values: dihedral angles from $2.4^{\circ}$ up to $56.5^{\circ}$, a twist angle between the two carbon rings of $45.5^{\circ}$ and $\mathrm{N} \cdots \mathrm{H}$ hydrogen bonds in the dimer with $\mathrm{N}$...H distances of $1.99 \AA$.

Table 1. Data collection and handling.

\begin{tabular}{ll}
\hline Crystal: & pale yellow prism, \\
& size $0.15 \times 0.15 \times 0.15 \mathrm{~mm}$ \\
Wavelength: & Mo $K_{\alpha}$ radiation $(0.71073 \AA)$ \\
$\mu:$ & $0.74 \mathrm{~cm}^{-1}$ \\
Diffractometer, scan mode: & Nonius Kappa CCD, $\varphi / \omega$ \\
$2 \theta_{\max }:$ & $53.98^{\circ}$ \\
$N(h k l)_{\text {measured }}, N(h k l)_{\text {unique: }}:$ & 5012,2615 \\
Criterion for $I_{\text {obs, }} N(h k l)_{\text {gt: }}:$ & $I_{\text {obs }}>2 \sigma\left(I_{\text {obs }}\right), 2149$ \\
$N(\text { param })_{\text {refined: }}$ & 213 \\
Programs: & SHELXS-97 [1], SHELXL-97 [2], \\
& SCHAKAL 99 [3]
\end{tabular}

Table 2. Atomic coordinates and displacement parameters (in $\AA^{2}$ ).

\begin{tabular}{llllll}
\hline Atom & Site & $x$ & $y$ & $z$ & $U_{\text {iso }}$ \\
\hline H(3) & $2 i$ & $0.232(2)$ & $0.903(2)$ & $-0.123(1)$ & $0.052(4)$ \\
H(4A) & $2 i$ & $0.060(3)$ & $0.821(2)$ & $0.088(2)$ & $0.072(5)$ \\
H(4B) & $2 i$ & $0.199(3)$ & $0.947(2)$ & $0.086(2)$ & $0.069(5)$ \\
H(5A) & $2 i$ & $0.282(2)$ & $0.586(2)$ & $0.196(2)$ & $0.071(5)$ \\
H(5B) & $2 i$ & $0.259(3)$ & $0.717(2)$ & $0.273(2)$ & $0.077(5)$ \\
H(6A) & $2 i$ & $0.610(2)$ & $0.616(2)$ & $0.238(2)$ & $0.060(4)$ \\
H(6B) & $2 i$ & $0.572(3)$ & $0.811(2)$ & $0.165(2)$ & $0.077(5)$ \\
H(7A) & $2 i$ & $0.492(3)$ & $0.644(2)$ & $-0.520(2)$ & $0.083(5)$ \\
H(7B) & $2 i$ & $0.292(3)$ & $0.674(2)$ & $-0.429(2)$ & $0.091(6)$ \\
H(7C) & $2 i$ & $0.378(3)$ & $0.821(3)$ & $-0.553(2)$ & $0.101(7)$ \\
H(8A) & $2 i$ & $0.843(3)$ & $0.703(3)$ & $-0.592(2)$ & $0.104(7)$ \\
H(8B) & $2 i$ & $0.973(4)$ & $0.848(3)$ & $-0.607(2)$ & $0.126(8)$ \\
H(8C) & $2 i$ & $0.741(4)$ & $0.888(3)$ & $-0.646(2)$ & $0.121(8)$ \\
H(2') & $2 i$ & $0.342(2)$ & $0.711(2)$ & $-0.242(1)$ & $0.048(3)$ \\
H(5') & $2 i$ & $0.998(3)$ & $0.864(2)$ & $-0.405(2)$ & $0.065(4)$ \\
H(6') & $2 i$ & $0.866(2)$ & $0.845(2)$ & $-0.195(2)$ & $0.059(4)$ \\
HO & $2 i$ & $1.015(3)$ & $0.477(2)$ & $0.102(2)$ & $0.088(6)$ \\
& & & & & \\
\hline
\end{tabular}

* Correspondence author (e-mail: johann.lex@uni-koeln.de) 
Table 3. Atomic coordinates and displacement parameters (in $\AA^{2}$ ).

\begin{tabular}{|c|c|c|c|c|c|c|c|c|c|c|}
\hline Atom & Site & $x$ & $y$ & $z$ & $U_{11}$ & $U_{22}$ & $U_{33}$ & $U_{12}$ & $U_{13}$ & $U_{23}$ \\
\hline$C(1)$ & $2 i$ & $0.6250(2)$ & $0.6839(1)$ & $0.0429(1)$ & $0.0378(6)$ & $0.0439(6)$ & $0.0361(6)$ & $-0.0044(5)$ & $-0.0021(4)$ & $-0.0150(5)$ \\
\hline$C(2)$ & $2 i$ & $0.5014(2)$ & $0.7685(1)$ & $-0.0665(1)$ & $0.0401(6)$ & $0.0399(6)$ & $0.0365(6)$ & $-0.0018(4)$ & $-0.0034(4)$ & $-0.0136(5)$ \\
\hline$C(3)$ & $2 i$ & $0.3110(2)$ & $0.8392(2)$ & $-0.0462(1)$ & $0.0451(7)$ & $0.0488(7)$ & $0.0443(7)$ & $0.0045(5)$ & $-0.0065(5)$ & $-0.0161(5)$ \\
\hline $\mathrm{C}(4)$ & $2 i$ & $0.2059(2)$ & $0.8351(2)$ & $0.0817(1)$ & $0.0446(7)$ & $0.0630(9)$ & $0.0534(8)$ & $0.0067(6)$ & $0.0008(6)$ & $-0.0262(6)$ \\
\hline $\mathrm{C}(5)$ & $2 i$ & $0.3117(2)$ & $0.7010(2)$ & $0.1908(1)$ & $0.0504(8)$ & $0.072(1)$ & $0.0428(7)$ & $0.0024(6)$ & $0.0034(6)$ & $-0.0218(7)$ \\
\hline$C(6)$ & $2 i$ & $0.5402(2)$ & $0.7029(2)$ & $0.1686(1)$ & $0.0497(7)$ & $0.0671(9)$ & $0.0411(7)$ & $0.0011(6)$ & $-0.0050(5)$ & $-0.0257(6)$ \\
\hline$C\left(1^{\prime}\right)$ & $2 i$ & $0.5895(2)$ & $0.7774(1)$ & $-0.1979(1)$ & $0.0416(6)$ & $0.0392(6)$ & $0.0352(6)$ & $0.0028(4)$ & $-0.0053(4)$ & $-0.0102(4)$ \\
\hline$C\left(2^{\prime}\right)$ & $2 i$ & $0.4759(2)$ & $0.7461(2)$ & $-0.2766(1)$ & $0.0422(6)$ & $0.0467(7)$ & $0.0429(6)$ & $0.0023(5)$ & $-0.007 !(5)$ & $-0.0158(5)$ \\
\hline$C\left(3^{\prime}\right)$ & $2 i$ & $0.5493(2)$ & $0.7566(2)$ & $-0.4012(1)$ & $0.0551(7)$ & $0.0452(7)$ & $0.0406(6)$ & $0.0086(5)$ & $-0.0124(5)$ & $-0.0163(5)$ \\
\hline$C\left(4^{\prime}\right)$ & $2 i$ & $0.7445(2)$ & $0.7997(2)$ & $-0.4495(1)$ & $0.0594(8)$ & $0.0522(7)$ & $0.0365(6)$ & $0.0044(6)$ & $-0.0017(5)$ & $-0.0128(5)$ \\
\hline$C\left(5^{\prime}\right)$ & $2 i$ & $0.8571(2)$ & $0.8318(2)$ & $-0.3713(1)$ & $0.0500(7)$ & $0.0617(8)$ & $0.0425(7)$ & $-0.0067(6)$ & $0.0027(6)$ & $-0.0119(6)$ \\
\hline$C\left(6^{\prime}\right)$ & $2 i$ & $0.7824(2)$ & $0.8216(2)$ & $-0.2479(1)$ & $0.0469(7)$ & $0.0522(7)$ & $0.0394(6)$ & $-0.0068(5)$ & $-0.0041(5)$ & $-0.0123(5)$ \\
\hline$C\left(7^{\prime}\right)$ & $2 i$ & $0.4192(3)$ & $0.7219(3)$ & $-0.4818(2)$ & $0.074(1)$ & $0.079(1)$ & $0.0577(9)$ & $0.0041(9)$ & $-0.0198(8)$ & $-0.035](9)$ \\
\hline$C\left(8^{\prime}\right)$ & $2 i$ & $0.8327(3)$ & $0.8109(3)$ & $-0.5833(2)$ & $0.087(1)$ & $0.095(1)$ & $0.0439(8)$ & $-0.005(1)$ & $0.0064(8)$ & $-0.0269(9)$ \\
\hline $\mathbf{N}$ & $2 i$ & $0.7965(1)$ & $0.5996(1)$ & $0.02391(9)$ & $0.0371(5)$ & $0.0523(6)$ & $0.0361(5)$ & $-0.0011(4)$ & $-0.0066(4)$ & $-0.0125(4)$ \\
\hline o & $2 i$ & $0.9015(1)$ & $0.5252(1)$ & $0.13440(9)$ & $0.0415(5)$ & $0.0763(7)$ & $0.0408(5)$ & $0.0056(4)$ & $-0.0114(4)$ & $-0.0170(4)$ \\
\hline
\end{tabular}

\section{References}

1. Meenakshi, C.; Ramamoorthy,V.; Muthusubramanian, S.; Sivasubramanian, S.; Ziegler, T.; Lex, J.: Crystal structure of 2-phenyl-2-cyclohexene-1-one oxime, $\mathrm{C}_{12} \mathrm{H}_{13} \mathrm{NO}$. Z. Kristallogr.NCS 216 (2001) 649-650.

2. Meenakshi, C.; Ramamoorthy,V.; Muthusubramanian, S.; Sivasubramanian, S.; Ziegler, T.; Lex, J.: Crystal structure of 2-(4-methoxyphenyl)-2cyclohexene-1-one oxime, $\mathrm{C}_{13} \mathrm{H}_{15} \mathrm{NO}_{2}$. Z. Kristallogr. NCS 216 (2001) 651-652.
3. Sheldrick, G. M.: SHELXS-97. Program for the Solution of Crystal Structures. University of Göttingen, Germany 1997.

4. Sheldrick, G. M.: SHELXL-97. Program for the Refinement of Crystal Structures. University of Göttingen, Germany 1997.

5. Keller, E.: SCHAKAL 99. University of Freiburg, Germany 1999. 